\title{
Structurally Controlled Bio-hybrid Materials Based on Unidirectional Association of Anisotropic Microparticles with Human Endothelial Cells
}

\author{
By Mutsumi Yoshida, Kyung-Ho Roh, Suparna Mandal, Srijanani Bhaskar, \\ Dongwoo Lim, Himabindu Nandivada, Xiaopei Deng, and Joerg Lahann*
}

In recent decades, biomedical researchers have increasingly realized the importance of local interactions between synthetic materials and cells for their macroscopic functionality. ${ }^{[1-3]}$ The groundbreaking progress in the biological sciences has led to a push towards custom-tailored biomaterials with precisely designed physico-chemical properties at the nano- and microscale. ${ }^{[3-5]}$ To date, a range of different approaches towards custom-tailored biomaterials exist based on different types of materials, such as hydrogels, ${ }^{[6,7]}$ microfabricated biomaterials, ${ }^{[8-11]}$ or electrospun fiber scaffolds. ${ }^{[12-19]}$ In addition, so-called bio-hybrid materials have been proposed, ${ }^{[20]}$ where cells and synthetic building blocks form structurally and functionally integrated architectures. ${ }^{[20-24]}$ Bio-hybrid materials may have important advantages over conventional biomaterials including improved cellular integration, potentially more straightforward in vivo applicability, or the ability to effectively remodel their materials structure with time. While bio-hybrid materials have so far been realized mostly as 2D cell sheets, ${ }^{[20-22]}$ a next generation of integrated biomaterials may be contemplated, where both synthetic building blocks, such as microor nanoparticles, and cells become integral structural elements that ultimately form the actual biomaterial through directional selfassembly in all three dimensions. ${ }^{[23-24]}$ In fact, entirely different biomaterials may be created based on well-ordered assemblies of cells and synthetic building blocks, as long as the synthetic building blocks can be designed to undergo spatially directed association with cells. In this respect, novel nano- and microparticle designs will have to be realized that are structurally anisotropic and, at the same time, show spatially-controlled affinity towards cells. When designing synthetic building blocks for such bio-hybrid materials, not only the chemical make-up of bulk and interfaces have been appreciated as important design criteria for biomaterials but also

[*] Prof. J. Lahann, Dr. M. Yoshida, Dr. S. Mandal, Dr. D. Lim, H. Nandivada Department of Chemical Engineering

The University of Michigan

3414 GGBL, 2300 Hayward

Ann Arbor, MI 4809

E-mail: lahann@umich.edu

Prof. J. Lahann, Dr. K.-H. Roh, S. Bhaskar, X. Deng

Macromolecular Science and Engineering

The University of Michigan

Ann Arbor, MI 4809

Prof. J. Lahann

Department of Materials Science and Engineering

The University of Michigan

Ann Arbor, MI 4809

DOI: 10.1002/adma.200901971 physical properties, such as microstructure, anisotropic particle architecture, or shape. ${ }^{[3]}$ While the potential of sophisticated bio-hybrid materials may be vast, with applications in areas ranging from novel actuators to tissue engineering and medical imaging, actual implementation has often been impeded by the lack of appropriate synthetic routes towards anisotropic building blocks. Only recently, a range of synthetic concepts has been developed for the fabrication of anisotropic particles, ${ }^{[25]}$ including microfluidic technologies, ${ }^{[26-30]}$ microfabrication, ${ }^{[31]}$ continuousflow polymerization, ${ }^{\left[{ }^{[2]}\right.}$ self-assembly, ${ }^{[33]}$ or electrohydrodynamic co-jetting. ${ }^{[34-39]}$

Herein, we report initial work towards a novel type of microstructured bio-hybrid material comprised of anisotropic polymer particles with spatially-controlled affinity towards human endothelial cells (Scheme 1a). These anisotropic particles consist of two distinct compartments that can exhibit vastly different functions. Here, functional bipolarity is realized by selective surface modification to create polymer particles with two biologically distinguishable hemispheres: one exhibiting high binding affinity for human endothelial cells and the other being essentially resistant towards cell binding (Scheme 1).

Bicompartmental particles were synthesized by simultaneous electrohydrodynamic co-jetting of two different solutions of polyacrylamide/poly(acrylic acid) co-polymers (PAAm-co-AA, Scheme 1b). While the base polymers were generally the same for both hemispheres, different functional groups were selectively incorporated into compartments to enable subsequent surface modifications. For instance, PAAm-co-AA polymers modified with biotin were used as components of one of the jetting solutions, when subsequent surface modification with streptavidin (SAv) was intended. The biotin-modified PAAm-co-AA polymer was obtained in a simple one-step synthesis. Carboxylic acid groups of PAAm-co-AA were activated using 1-ethyl-3-(3-dimethylaminopropyl)carbodiimide hydrochloride in combination with $N$-hydroxysulfo-succinimide sodium salt (EDC/sulfo-NHS) as coupling agents. After the reaction, excess reagent and urea were removed by dialysis and the final product was lyophilized and characterized by ${ }^{1} \mathrm{H}$-NMR spectroscopy (Supporting Information, Fig. S1). Alternatively, acetylene-modified PAAm-co-AA was added to the jetting solutions, when compartment-specific surface modification via copper(I)-catalyzed Huisgen 1,3-dipolar cycloaddition was intended. For this purpose, acetylene-modified PAAm-co-AA was prepared using a method similar to the one used for biotin modification of PAAm-co-AA. Again, free acid groups were activated in the presence of EDC/sulfo-NHS and were reacted with propargyl amine at room temperature for four 


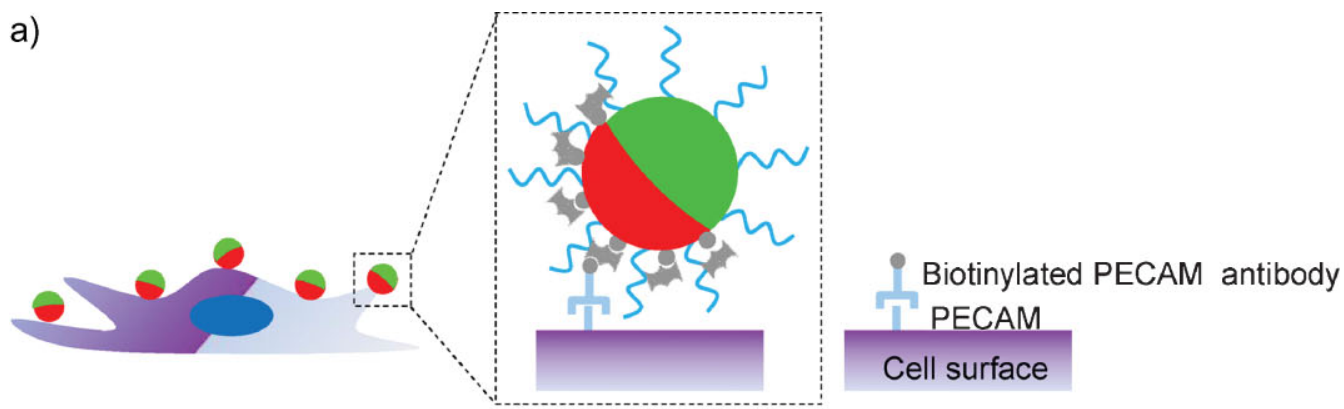

b)

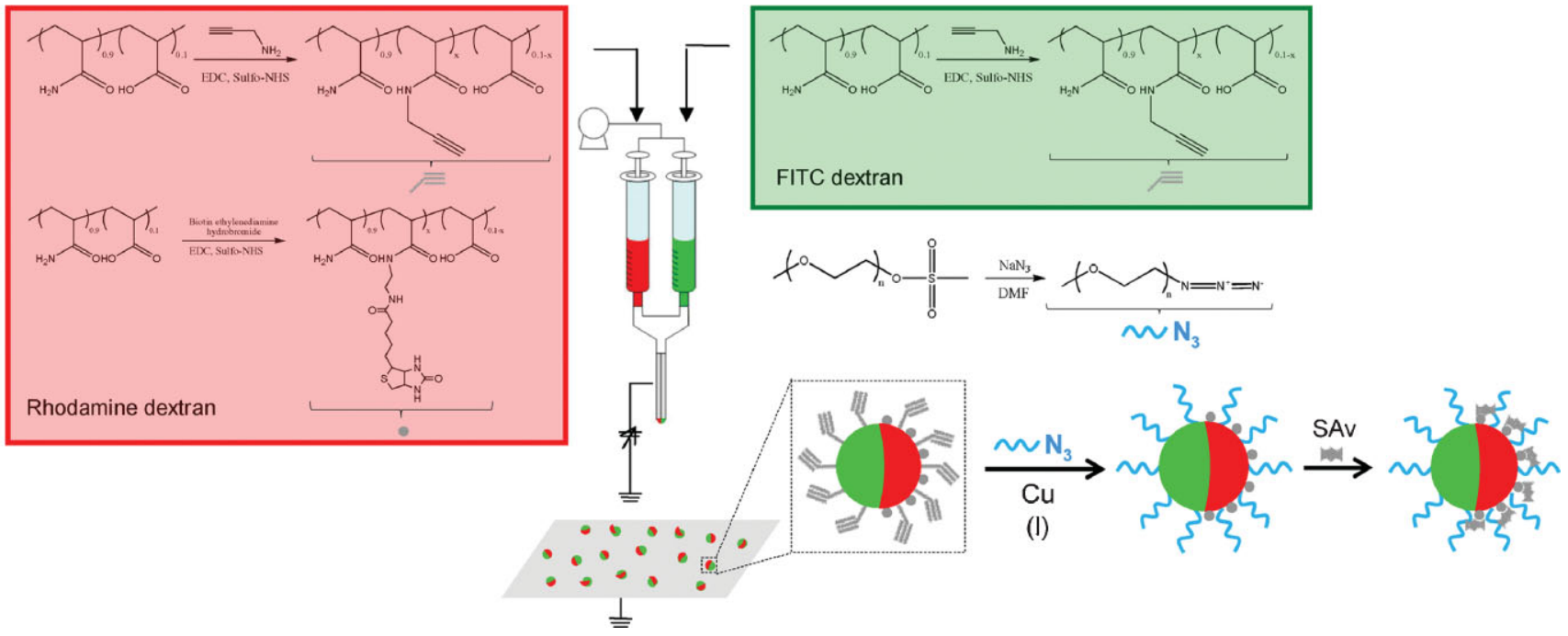

Scheme 1. Synthesis and unidirectional self-assembly of bicompartmental particles. a) Scheme for the binding of bicompartmental particles to cells via streptavidin present on the particle surface of one compartment and biotin from cell-specific antibody (in this case PECAM). b) Bicompartmental particles were fabricated via electrohydrodynamic co-jetting of polymer solutions. Reaction schemes for the incorporation of PEG and streptavidin are shown as examples.

hours, as confirmed by ${ }^{1} \mathrm{H}-\mathrm{NMR}$ spectroscopy (Supporting Information, Fig. S1). Moreover, fluorescein isothiocyanate (FITC)- or rhodamine-conjugated dextran were selectively added to the jetting solutions to confirm particle anisotropy or to monitor unidirectional association between particles and cells using fluorescence imaging (Scheme 1a). ${ }^{[34]}$

In general, electrohydrodynamic co-jetting of two different PAAm-co-AA solutions yielded particles with well-defined, spherical morphologies. Figure 1a shows a confocal laser scanning microscopy (CLSM) image of a typical batch of bicompartmental particles suspended in aqueous solution. The CLSM image reveals confinement of green and red fluorescence into individual hemispheres, suggesting the presence of two distinct compartments. The presence of distinct compartments was further supported by quantitative analysis of a representative population of bicompartmental particles via flow cytometry (Fig. 1b). In this experiment, bicompartmental particles containing Atto 665 in one compartment and FITC in the other compartment were compared to bicompartmental particles without the fluorochromes or those with either Atto 665 or FITC alone in one of the compartments. For bicompartmental particles loaded with both fluorochromes, more than $95 \%$ of the particle population was localized in the upper right quadrant, indicating that the particles were indeed positive for both Atto 665 and FITC (Fig. 1b,c). This result was in distinct difference to the remaining three particle populations, which were either positive for Atto 665 or FITC only or did not contain any fluorochrome (lower left quadrant). The flow cytometric data are in excellent agreement with the bicompartmental nature of the particles, as observed by CLSM (Fig. 1a), and were in general agreement with previously reported bicompartmental particles. ${ }^{[34]}$ To stabilize the particles for subsequent surface modification and use in physiological media, bicompartmental particles were crosslinked via thermal imidization, ${ }^{[36]}$ which resulted in particles that formed a well-dispersed suspension and were stable over several weeks. After surface modification with polyethylene glycol (PEG) and streptavidin, the bicompartmental particle suspensions were examined by CLSM. A typical CLSM image of an aqueous particle suspension $(\mathrm{pH}=7.4)$ is shown in Figure $\mathrm{S} 2$ of the Supporting Information. Image analysis revealed an average hydrodynamic radius of $847 \mathrm{~nm}$ and a standard deviation of $478 \mathrm{~nm}$ for the particles shown in Figure S2. This is higher than comparable bicompartmental particles without surface modification, which had an average radius of $427 \pm 234 \mathrm{~nm}$, determined by dynamic light scattering. ${ }^{[36]}$

Prior to studying directed interactions of bicompartmental particles with human endothelial cells, the initial biocompatibility of these particles was evaluated. Human umbilical vein endothelial cells (HUVECs) were incubated with non-modified bicompartmental PAAm-co-AA particles or those that were surface-modified 
a)
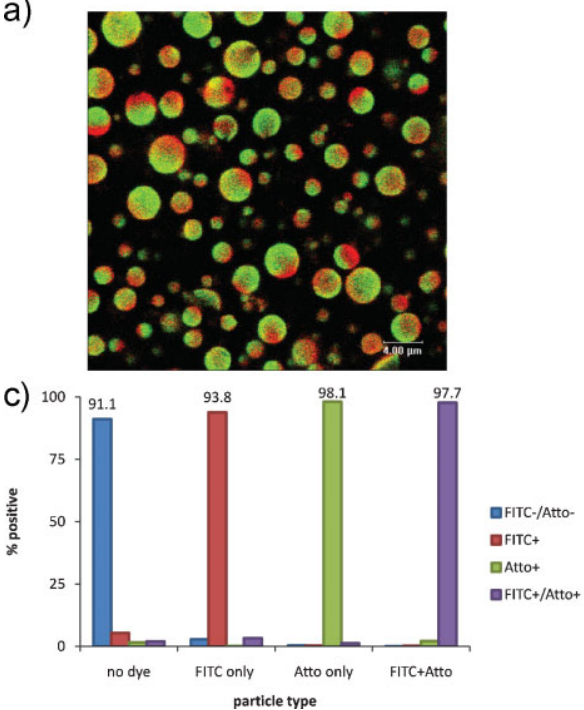

b)
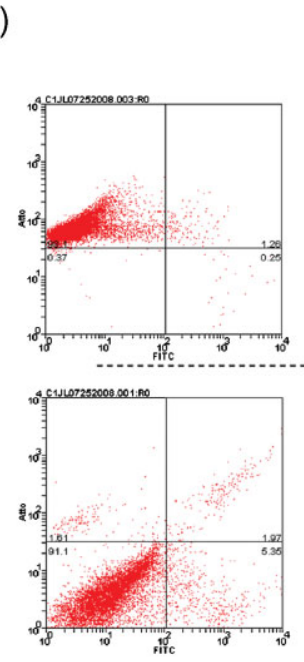
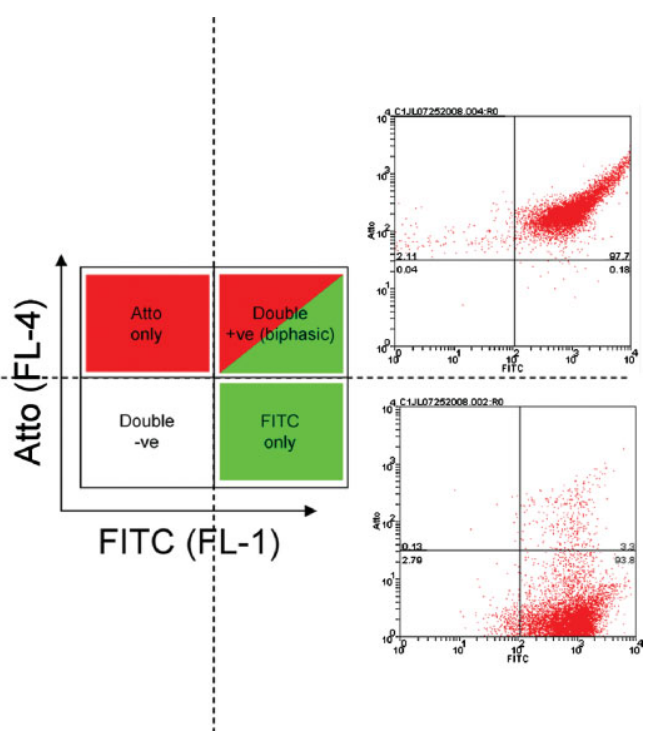

Figure 1. Particle characterization. a) CLSM image of bicompartment particles presenting PEG on both compartments (red and green) and streptavidin in the compartment including rhodamine dextran (red). b) Flow cytometry analysis of bicompartmental particles. c) Graphical representation of the flow cytometry data shown in (b). Further details are provided in the Supporting Information, Figure S3.

with either PEG or biotin/streptavidin for 24 or 96 hours at various concentrations. The presence of the particles did not significantly affect the proliferation of HUVECs, as determined by a standard MTS assay [3-(4,5- dimethylthiazol-2- yl)-5-(3-carboxymethoxyphenyl)-2-(4-sulfophenyl)-2H-tetrazolium] (Fig. 2a). Similarly, cell viability evaluated using Annexin V and propidium iodide (PI) as markers of cell apoptosis and death, respectively, revealed no statistically significant difference in the total percentage of live cell population after exposure to particles, as compared to cells grown in the absence of particles. These initial biocompatibility experiments suggest that the herein developed bicompartmental microparticles do not appear to exhibit significant cytotoxic effects that would result in a decrease of cell proliferation and viability. In fact, the data obtained for surface-modified PAAm-co-AA particles are in general agreement with a previous analysis of short-term biocompatibility of bicompartmental particles ${ }^{[37]}$ and confirm that the introduction of bicompartmental particles to HUVECs may be considered safe, at least in vitro, within the particle doses and incubation times pursued herein to form bio-hybrid materials.

To demonstrate compartment-specific association between bicompartmental particles and human cells, particles prepared by electrohydrodynamic co-jetting were selectively modified with streptavidin in one hemisphere only. The selective incorporation of biotin in one compartment was confirmed by self-assembly of biotin with fluorescence-labeled streptavidin and subsequent CLSM analysis (Supporting Information, Fig. S3). These particles were then incubated under serum-free conditions with human endothelial cells that were labeled with either biotinylated antibody against platelet endothelial cell-adhesion molecule (PECAM), which is highly expressed on endothelial cells including HUVECs, or with a biotinylated isotype antibody, which exhibits only low-level, non-specific binding to the cells. This preparation was pursued to ensure introduction of a biotin linker, which binds to streptavidin with exceptionally high affinity, thereby effectively coupling cells and particles (Scheme 1). We further confirmed the controlled binding of particles in the presence of serum, since serum contains proteins that potentially may interfere with the ability to undergo self-assembly. In a control experiment, monocompartmental PAAm-co-AA particles (i.e., particles with one single compartment) were prepared by electrohydrodynamic jetting, which contained $13 \%(\mathrm{w} / \mathrm{w})$ of the biotin-modified PAAm-co-AA polymer, and were allowed to contact surfaces that presented the complementary binding molecule streptavidin. To prepare these surfaces, we used chemical vapor deposition (CVD) polymerization, which has been used previously for deposition of reactive coatings onto flat and $3 \mathrm{D}$ substrates. ${ }^{[40-42]}$ In this case, we used CVD polymerization to deposit approximately 50-nm-thick films of poly(4-heptadecafluorononanoyl- $p$-xylylene-co- $p$-xylelene), followed by immobilization of biotin-hydrazide and streptavidin. ${ }^{[43]}$ The streptavidin-presenting substrates were incubated with particles under serum-containing conditions (2\% fetal bovine serum, FBS). Particles without biotin ligands were used as a reference. As shown in Figure S5 of the Supporting Information, biotin-containing particles but not biotin-free particles bound to the streptavidin-modified surfaces. The presence of serum did not prevent the ability of the particles to bind to the surface as indicated by the increase in red color for the biotin-containing particles.

Finally, particle binding to cells was assessed by flow cytometry as the increase in cell-associated FITC fluorescence, which is caused by cell/particle association. Initial experiments revealed substantial non-specific binding between bicompartmental particles modified with streptavidin in one compartment and human endothelial cells. This non-specific binding was present also with non-modified bicompartmental particles, regardless of whether the cells were stained with endothelial-cell-specific or with non-specific control antibodies (Fig. 3a). The non-specific binding established a serious challenge to further steps towards the design of well-defined bio-hybrid materials, as the preparation of structurally controlled bio-hybrid materials require high selectivity between binding and non-binding particle compartments. To mitigate the non-specific binding of particles to cells, the entire particle surface, i.e., the surface of both compartments, was coated with PEG via 

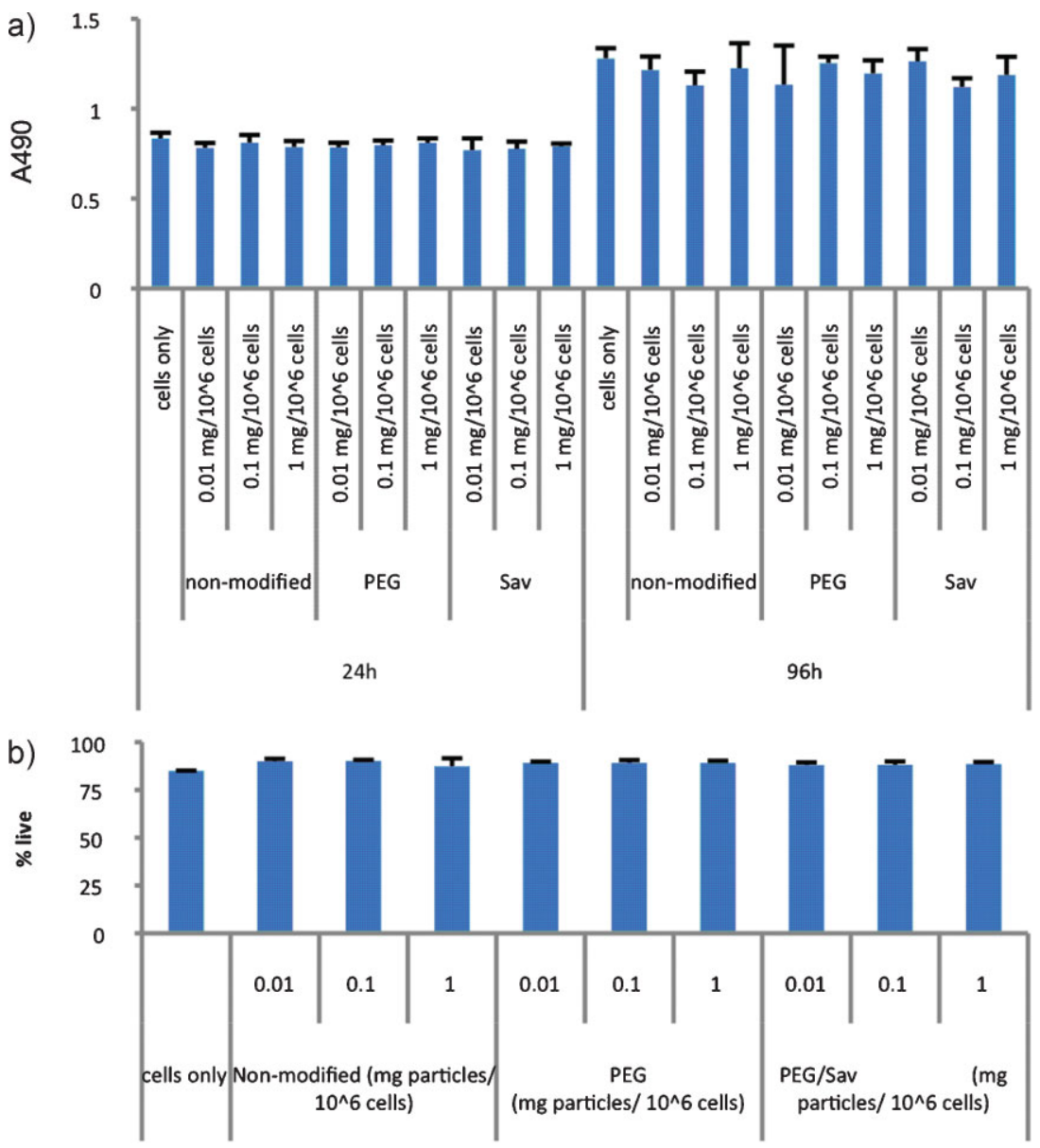

Figure 2. Short-term biocompatibility of bicompartmental particles with various surface modifications. a) Effect of bicompartmental particles on HUVEC proliferation as measured by a colorimetric MTS assay. b) Effect of bicompartmental particles on HUVEC viability as assessed by flow cytometry via Annexin V/PI labeling.

copper(I)-catalyzed Huisgen 1,3-dipolar cycloaddition. The coupling reaction occurred between acetylene groups of acetylenemodified PAAm-co-AA added to both jetting solutions and azide groups introduced to PEG prior to surface coupling. ${ }^{[9,10]}$ As expected, modification of the particle surface via immobilization of PEG significantly decreased non-specific association between particles and cells (Fig. 3a). Moreover, streptavidin-modified particles bound specifically to HUVECs that were labeled with antibody against PECAM but not to cells labeled with the isotype control antibody. The fluorescence signal for cells incubated with the non-specific isotope antibody was not significantly different from cell autofluorescence observed with cells in the absence of particles. This strongly supports unidirectional association between anisotropic particles and human endothelial cells. The flow cytometric analysis was further supported by CLSM (Fig. 3b). For this purpose, cells bound to particles were separated from free particles by fluorescence activated cell sorting. The overlay of differential interference contrast and fluorescent images corroborate increased cellular binding observed with streptavidin-modified particles as compared to particles that were not modified or presented PEG alone.

When incubated with human endothelial cells, these particles exhibit a bipolar behavior, where one compartment exhibits binding affinity towards human endothelial cells, while the other compartment was essentially non-binding (Fig. 3). In this design, particles not only bound to the top of the human endothelial cells, but associated all around the perimeter of cells forming a single particle lining (Fig. 4).

With the fundamental feasibility of anisotropic particles for self-association with cells demonstrated, future work will need to diversify on the type of anisotropic building blocks that can be designed for use in bio-hybrid materials. An essential requirement for bio-hybrid materials will be the availability of synthetic building blocks that can provide directionally controlled binding patterns at cellular or subcellular resolution. Specifically, controlling the number of compartments, compartment sizes, the relative positioning of compartments on a particle, or the introduction of differently shaped building blocks, such as bicompartmental rods, will enable the synthesis of many novel bio-hybrid architectures. Such novel type of biomaterials may be of equal importance for medical as well as biotechnological applications including regenerative medicine, medical imaging, and the design of novel actuators or power harvesting and storage devices.

\section{Experimental}

Materials: Poly(acrylamide-co-acrylic acid, sodium salts) (PAAm-co-AA) (Mw $200 \mathrm{kD}, 10 \%$ acrylic acid residues) was purchased from Polysciences (Warrington, PA). Polyethylene glycol monomethyl ether mesylate (methyl-PEG-mesylate, $\mathrm{M}_{w} 5 \mathrm{kD}$, Fluka) and sodium azide (Sigma) were dissolved in dimethylformamide (DMF) and stirred at $60^{\circ} \mathrm{C}$ for $24 \mathrm{~h}$. The polymer product was precipitated by addition of excess ethyl ether and recovered by filtration. The filtrate was dissolved in water and dialyzed (membrane molecular weight cut off is $3 \mathrm{kD}$ ) overnight and subsequently lyophilized.

Fabrication of Bicompartmental Particles via Electrohydrodynamic co-jetting: Bicompartmental PAAm particles were prepared by electrohydrodynamic co-jetting as previously described [34,36]. All components were dissolved in double distilled (dd) $\mathrm{H}_{2} \mathrm{O}$ and reported as weight per volume. Particles presenting acetylene groups or biotin were prepared using the following jetting solutions, respectively, with $0.5 \%$ FITC- or rhodamine-conjugated dextran: 4\% PAAm-co-AA and 1\% acetylene-modified PAAm-co-AA or 3\% PAAm-co-AA, $1 \%$ acetylene-modified PAAm-co-AA, and $1 \%$ biotin-modified PAAm-co-AA. Following the electrohydrodynamic co-jetting, particles were incubated at $175^{\circ} \mathrm{C}$ for $3 \mathrm{~h}$ to induce thermal imidization between carboxylic acid and amide groups.

Surface Modification of Bicompartmental Particles: Particles were modified with PEG using a copper(I)-catalyzed Huisgen 1,3-dipolar cycloaddition. Briefly, bicompartmental particles were suspended in dd $\mathrm{H}_{2} \mathrm{O}$, to which methyl-PEG-azide was added. $0.3 \mathrm{~m}$ sodium ascorbate and $0.03 \mathrm{M} \mathrm{CuSO}_{4} \cdot \mathrm{H}_{2} \mathrm{O}$ were added to the suspension, and the mixture was maintained on a hematology mixer at room temperature for $10 \mathrm{~h}$. Resulting particles were washed 3 times with $d d \mathrm{H}_{2} \mathrm{O}$ by centrifugation and finally resuspended in phosphate buffered saline (PBS). Next, PEG-modified particles prepared from jetting solution containing biotin-modified 
a)

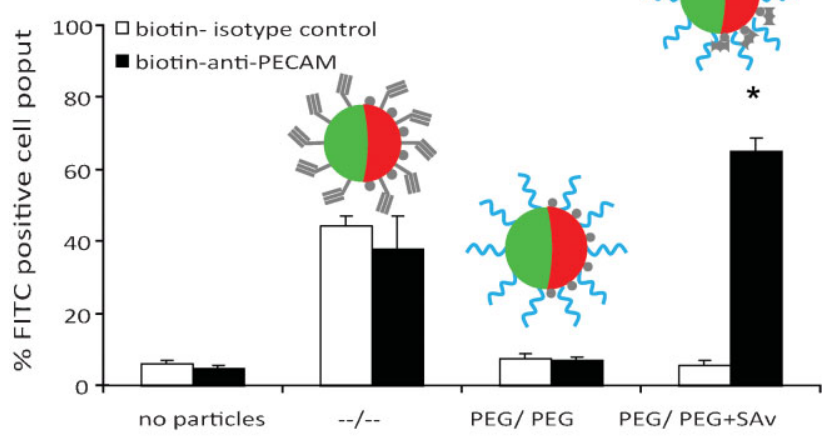

b)
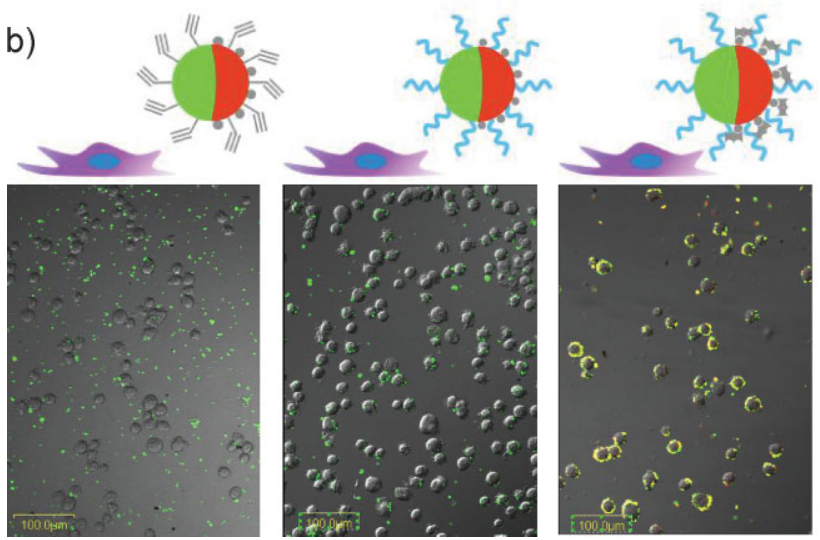

Figure 3. Directional cell binding of bicompartmental particles. a) Quantification of the percent of cell population with bound bicompartmental particles and corresponding CLSM images. -/-: unmodified bicompartmental particles, PEG/PEG: bicompartment particles with PEG on the surface of both compartments, PEG/PEG + SAv: bicompartment particles with PEG on surface of both compartments with streptavidin (SAv) only on the surface of one compartment. b) Overlays of CLSM images with differential interference contrast images corresponding to the samples studied by flow cytometry. All scale bars are $100 \mu \mathrm{m}$.

PAAm-co-PAA were suspended in PBS and incubated with streptavidin (Pierce, Rockford, IL) for $6 \mathrm{~h}$ at room temperature on a hematology mixer. The final suspension was washed with fresh PBS 3 times and isolated by centrifugation.

Characterization of Bicompartmental Particles: For imaging of the particles by CLSM (SP2 CLSM, Leica, USA), a suspension of bicompartmental particles in water was placed between two cover slips. FITC and rhodamine were excited with Ar/ArKr or GreNe laser at $488 \mathrm{~nm}$ or $543 \mathrm{~nm}$, respectively. Typical images of bicompartmental particle suspensions were analyzed by Image J [44] to obtain size-distribution measurements (Supporting Information, Figure S2). The errors in particle recognition by Image I were corrected for individual particles by visual comparison with the original image and the surface area of individual particle was converted to the diameter assuming a perfectly spherical envelope.

Bicompartmental character of the particles was analyzed by flow cytometry as previously described [36]. To minimize any bleed-over of fluorescent dyes, FITC- and Atto 655-conjugated dextrans were used. Particles without any fluorochrome, those loaded with either FITC- or Atto 655-dextran alone, or those with both fluorochrome in each compartment were resuspended in PBS and analyzed using fluorescence-activated cell sorting (FACSCalibur, Becton Dickinson Biosciences, San Jose, CA) at the University of Michigan Flow Cytometry Core Facility. For each sample, 20000 events were collected and fluorescence signals from FITC- a)

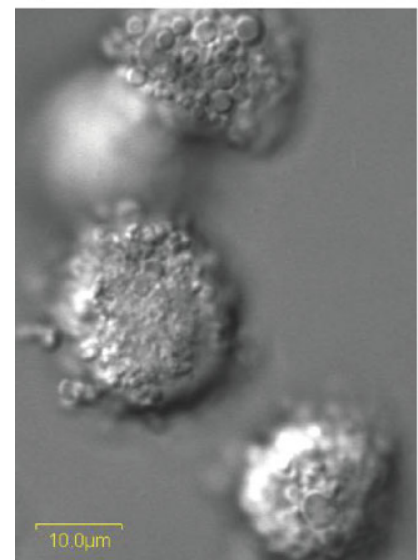

c)

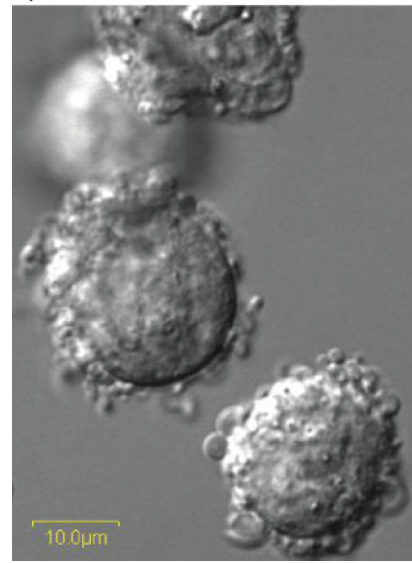

d)
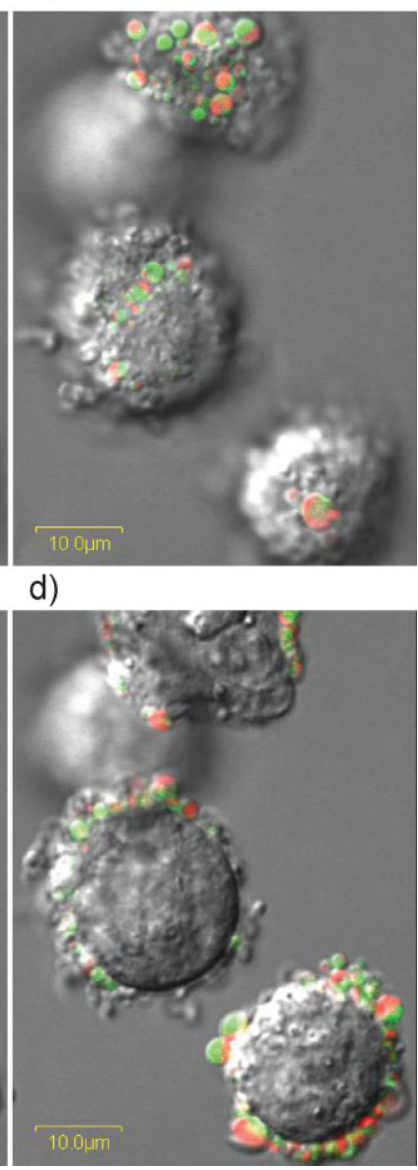

Figure 4. Self-assembly of bicompartmental particles and human endothelial cells. a,c) CLSM images and b,d) overlays of CLSM images with differential interference contrast images of human endothelial cells selfassembled with PEG/PEG + SAv particles. Images were taken within the same field of view at two different focal planes towards the top of the cells $(a, b)$ and at half cell height $(c, d)$. All scale bars are $10 \mu \mathrm{m}$.

and Atto 655-conjugated dextrans were resolved in FL1 (excitation with a $488 \mathrm{~nm}$ laser with $530 / 30$ bandpass filter) and FL4 (excitation with a $635 \mathrm{~nm}$ laser with $661 / 16$ bandpass filter) channels, respectively. Data acquisition was performed using CellQuest Pro (BD Biosciences) and analysis was performed using WinMDI (Scripps Research Institute, La Jolla, CA, USA). Population within the first decade is considered to be negative for the fluorescence and the double positive population in the upper right corner is considered to be bicompartmental.

Short-Term Biocompatibility of Bicompartmental Particles: The effect of bicompartmental particles on HUVEC proliferation was evaluated using a commercially available colorimetric cell proliferation assay (CellTiter 96 Aqueous One Solution Cell Proliferation Assay, Promega, Madison, WI, USA) as previously reported [37]. Briefly, particles were added to cells cultured in Endothelial cell Growth Media containing 2\% FBS (Lonza, Basel, Switzerland) at a dose of $0.01,0.1$, or $1 \mathrm{mg}$ per $10^{6}$ initial number of cells seeded. At 4, 24, 48, 72, and $96 \mathrm{~h}$ after incubation with the bicompartmental particles, colorimetric reagent was added for $4 \mathrm{~h}$ and absorbance was measured at $490 \mathrm{~nm}$ using a spectrophotometric plate reader (Molecular Dynamics).

The degree of cytotoxicity of bicompartmental particles was assessed by viability staining using flow cytometry as previously described [37]. In brief, HUVECs cultured in Endothelial cell Growth Media containing 2\% FBS incubated with or without $0.01,0.1$, or $1 \mathrm{mg}$ bicompartmental nanocolloids 
(FITC only) per $10^{6}$ initially seeded cells for $24 \mathrm{~h}$ were stained with Alexa fluor 647-conjugated Annexin V and PI (Invitrogen) for $30 \mathrm{~min}$. Samples were analyzed by FACSCalibur. Cell population with increased fluorescence resolved in FL1 channel (excitation by $488 \mathrm{~nm}$ laser, 530/30 filter) was gated as cells with associated bicompartmental particles. Cell population that were double negative or positive for both Annexin $V$ (resolved in FL4, excitation by a $635 \mathrm{~nm}$ laser with 661/16 filter) and PI (resolved in FL2, excitation by a $488 \mathrm{~nm}$ laser with $585 / 42$ filter) were considered to be dead or alive, respectively.

Directional Binding of Bicompartmental Particles to Cells: Binding of bicompartmental particles to cells was first evaluated using flow cytometry. Human umbilical vein endothelial cells (HUVECs; $p 5$ ) suspended in $0.5 \%$ bovine serum albumin (BSA) in PBS were stained with biotin-conjugated anti-human IgC isotype control or anti-human CD31 antibody (Southern Biotech, Birmingham, AL) for $30 \mathrm{~min}$ at $4{ }^{\circ} \mathrm{C}$. Upon washing in ice-cold buffer (PBS $+0.5 \%$ BSA) 3 times by centrifugation, these cells were subsequently incubated with indicated particles $\left(0.5 \mathrm{mg}\right.$ per $10^{6}$ cells) for $30 \mathrm{~min}$ at $4{ }^{\circ} \mathrm{C}$ on hematology mixer, washed, and assessed by flow cytometry using a BD FACSCalibur (Becton Dickinson, Franklin Lakes, NJ) and reported as percent of cell population with bound particles.

In some cases, cells bound to particles were sorted to separate non-bound, free particles by fluorescence activated cell sorting using BD FACSDiVa (Becton Dickinson) equipped with 3 lasers $(488 \mathrm{~nm}, 350 \mathrm{~nm}$, and $633 \mathrm{~nm}$ ). Cell population before and after sorting were viewed using an Olympus FluoView 500 laser scanning confocal microscope (Olympus America, Center Valley, PA) equipped with a diode laser with UV excitation $(405 \mathrm{~nm})$, a multi-line argon laser $(458 \mathrm{~nm}, 488 \mathrm{~nm}$, and $515 \mathrm{~nm})$, and two solid-state Helium Neon lasers $(543 \mathrm{~nm}$ and $633 \mathrm{~nm}$ ).

Orientation of particle binding to cells was further visualized using CLSM. HUVECs grown on poly-L-lysine coated cover slips were rinsed 3 times with D-PBS, then fixed with $4 \%$ paraformaldehyde (Sigma) and permeabilized with $0.1 \%$ Triton X-100 (Sigma). Upon rinsing with PBS with $0.1 \%$ Tween20 (PBS-T), cells were blocked with $5 \%$ normal goat serum (NGS) (Zymed, Carlsbad, CA) for $1 \mathrm{~h}$. Cells were then stained with mouse anti-human PECAM (Chemicon, Billerica, MA) for $1 \mathrm{~h}$, followed by biotin-conjugated goat anti-mouse IgG1 (Caltag Laboratories, Carlsbad, CA) and in some cases also with Alexa 633-conjugated phalloidin for $30 \mathrm{~min}$. Cells were washed and subsequently incubated with indicated particle suspensions on a rotator for $30 \mathrm{~min}$ under serum-free conditions. Loosely or non-bound particles were washed with PBS-T. Cover slips were mounted with ProLong Gold and stained with 4',6-diamidino2-phenylindoldihydrochloride (DAPI, Invitrogen, Carlsbad, CA) and stored overnight at $4{ }^{\circ} \mathrm{C}$ and imaged using an Olympus FluoView 500 laser scanning confocal microscope (Olympus America).

Statistical Analysis: Statistical analysis was performed using a general linear model ANOVA with Minitab software (Version 15, Minitab, State College, PA, USA). Unless otherwise indicated, $p$-values of 0.05 were considered significant. Experiments were repeated as indicated.

\section{Acknowledgements}

M. Y. and K. H. R. contributed equally to this work. Supporting Information is available online from Wiley InterScience or from the author.

Received: June 12, 2009

Published online: September 18, 2009

[1] N. A. Peppas, R. Langer, Science 1994, 263, 1715.

[2] R. Langer, D. A. Tirrell, Nature 2004, 428, 487.

[3] S. Mitragotri, J. Lahann, Nat. Mater. 2009, 8, 15.
[4] M. Yoshida, R. Langer, A. Lendlein, J. Lahann, Polym. Rev. 2006, 46, 347.

[5] W. F. Liu, C. S. Chen, Adv. Drug Delivery Rev. 2007, 59, 1319.

[6] N. A. Peppas, J. Z. Hilt, A. Khademhosseini, R. Langer, Adv. Mater. 2006, 18, 1345.

[7] M. C. Cushing, K. S. Anseth, Science 2007, 316, 1133.

[8] V. L. Tsang, S. N. Bhatia, Adv. Drug Delivery Rev. 2004, 56, 1635.

[9] D. W. Hutmacher, J. Biomater. Sci. Polym. Ed. 2001, 12, 107.

[10] D. S. Kohane, R. Langer, Pediatr. Res. 2008, 63, 487.

[11] A. Khademhosseini, R. Langer, J. Borenstein, J. P. Vacanti, Proc. Natl. Acad. Sci. USA 2006, 103, 2480.

[12] M. Goldberg, R. Langer, X. Q. Jia, J. Biomater. Sci. Polym. Ed. 2007, 18, 241

[13] J. Venugopal, S. Low, A. T. Choon, S. Ramakrishna, J. Biomed. Mater. Res. B 2008, 84B, 34

[14] I. K. Kwon, S. Kidoaki, T. Matsuda, Biomaterials 2005, 26, 3929.

[15] F. Yang, R. Murugan, S. Wang, S. Ramakrishna, Biomaterials 2005, 26, 2603.

[16] S. Bhaskar, J. Lahann, J. Am. Chem. Soc. 2009, 131, 6650.

[17] S. Y. Chew, Y. Wen, Y. Dzenis, K. W. Leong, Curr. Pharm. Des. 2006, 12, 4751.

[18] J. Xie, X. Li, Y. Xia, Macromol. Rapid Commun. 2008, 29, 1775.

[19] T. J. Sill, H. A. von Recum, Biomaterials 2008, 29, 1989.

[20] A. W. Feinberg, A. Feigel, S. S. Shevkoplyas, S. Sheeky, G. M. Whiteside, K. K. Parker, Science 2007, 317, 1366.

[21] M. Ebara, M. Yamato, T. Aoyagi, A. Kikuchi, K. Sakai, T. Okano, Biomacromolecules 2004, 5, 505.

[22] S. Okajima, Y. Sakai, T. Yamaguchi, Langmuir 2005, 21, 4043.

[23] O. Kriha, M. Becker, M. Lehmann, D. Kriha, J. Kriegelstein, M. Yosef, S. Schlecht, R. B. Wehrspohn, J. H. Wendorff, A. Greiner, Adv. Mater. 2007, 18, 2483.

[24] A. J. Swiston, C. Cheng, S. H. Um, D. J. Irvine, R. E. Cohen, M. F. Rubner Nano Lett. 2008, 8, 4446.

[25] M. Yoshida, J. Lahann, ACS Nano 2008, 6, 1101.

[26] A. S. Utada, E. Lorenceau, D. R. Link, P. D. Kaplan, H. A. Stone, D. A. Weitz, Science 2005, 308, 537.

[27] S. Q. Xu, Z. H. Nie, M. Seo, P. Lewis, E. Kumacheva, H. A. Stone, P. Garstecki, D. B. Weibel, I. Gitlin, G. M. Whitesides, Angew. Chem. Int. Ed. 2005, 44, 724

[28] T. Nisisako, T. Torii, T. Takahashi, Y. Takizawa, Adv. Mater. 2006, 18, 1152.

[29] Z. H. Nie, W. Li, M. Seo, S. Q. Xu, E. Kumacheva, J. Am. Chem. Soc. 2006, $128,9408$.

[30] T. Nisikado, T. Torii, Lab on a chip 2008, 8, 287.

[31] J. P. Rolland, B. W. Maynor, L. E. Euliss, A. E. Exner, G. M. Denison, J. M. DeSimone, J. Am. Chem. Soc. 2005, 127, 10096.

[32] D. Dendukuri, D. C. Pregibon, J. Collins, T. A. Hatton, P. S. Doyle, Nat Mater. 2006, 5, 365

[33] A. Walther, K. Matussek, A. H. E. Mueller, Nano 2008, 2, 1167.

[34] K.-H. Roh, D. C. Martin, J. Lahann, Nat. Mater. 2005, 4, 759

[35] K.-H. Roh, D. C. Martin, J. Lahann, J. Am. Chem. Soc. 2006, 128, 6796.

[36] K.-H. Roh, M. Yoshida, J. Lahann, Langmuir 2007, 23, 5683.

[37] M. Yoshida, K.-H. Roh, J. Lahann, Biomaterials 2007, 28, 2446.

[38] S. Bhaskar, J. Hitt, S. W. L. Chang, J. Lahann, Angew. Chem. Int. Ed. 2009, $25,4589$.

[39] S. Bhaskar, K.-H. Roh, X. Jiang, G. A. L. Baker, J. Lahann, Macromol. Rapid Commun. 2008, 29, 1655.

[40] J. Lahann, Polym. Int. 2006, 55, 1361.

[41] H.-Y. Chen, Y. Elkasabi, J. Lahann, J. Am. Chem. Soc. 2006, 128, 374.

[42] H. Y. Chen, J. Lahann, Anal. Chem. 2005, 77, 6909.

[43] H. Nandivada, H. Y. Chen, J. Lahann, Macromol. Rapid Commun. 2005, 26, 1794.

[44] M. D. Abramoff, P. J. Magelhaes, S. J. Ram, Biophotonics Int. 2004, 11 (7), 36. 